\title{
Standard R-CHOP Therapy in Follicular Lymphoma and Diffuse Large B-Cell Lymphoma
}

\author{
Naoto Tomita, ${ }^{1)}$ Hirotaka Takasaki, ${ }^{2)}$ Shin Fujisawa, ${ }^{3)}$ Kazuho Miyashita, ${ }^{4)}$ Eriko Ogusa, ${ }^{5)}$ \\ Kumiko Kishimoto, ${ }^{6}$ Shiro Matsuura, ${ }^{7)}$ Rika Sakai, ${ }^{2}$ Hideyuki Koharazawa, ${ }^{3)}$ Wataru Yamamoto, ${ }^{2)}$ \\ Katsumichi Fujimaki, ${ }^{4)}$ Hiroyuki Fujita, ${ }^{1)}$ Yoshimi Ishii, ${ }^{2)}$ Jun Taguchi, ${ }^{5)}$ Hideyuki Kuwabara, ${ }^{3)}$ \\ Shigeki Motomura, ${ }^{2)}$ and Yoshiaki Ishigatsubo ${ }^{1)}$
}

The introduction of rituximab (R) has measurably improved the outcome of patients with follicular lymphoma (FL) and diffuse large B-cell lymphoma (DLBCL). To evaluate the outcome of patients with FL and DLBCL under R plus CHOP therapy, we performed a retrospective analysis in Yokohama City University Hematology Group in Japan. Five hundred and twenty-six patients $(158, \mathrm{FL} ; 368$, DLBCL) were scheduled to undergo primary therapy with 6 cycles of full-dose R-CHOP therapy with curative intent. The median observation periods in living patients with FL and DLBCL were 45 months and 43 months, respectively. The complete response, 5-year progression-free survival (PFS), and 5-year overall survival (OS) rates were $86 \%, 50 \%$, and $92 \%$ in the FL group, and $89 \%, 72 \%$, and $80 \%$ in the DLBCL group, respectively. Although PFS was significantly better in the DLBCL group than in the FL group, OS was significantly better in FL patients. We also found that the OS and PFS of grade $3 \mathrm{FL}$ patients were not statistically different from those with grade 1-2. These findings indicate that all grades of FL should be categorized simply as "FL" with regard to R-CHOP therapy. Our results also demonstrate the incurability of FL (grade 1-3B), even with R-CHOP therapy. 〔J Clin Exp Hematop 53(2) : 121-125, 2013]

Keywords: diffuse large B-cell lymphoma, follicular lymphoma, R-CHOP

\section{INTRODUCTION}

In almost all cases, follicular lymphoma (FL) and diffuse large B-cell lymphoma (DLBCL) are CD20-positive. Since the introduction of rituximab $(\mathrm{R})$ treatment, which targets $\mathrm{CD} 20$, the outcome of patients with $\mathrm{FL}^{1}$ or $\mathrm{DLBCL}^{2,3}$ has improved noticeably. Indeed, treatment with R-CHOP chemoimmunotherapy [ $50 \mathrm{mg} / \mathrm{m}^{2}$ doxorubicin (adriamycin) on day $1,750 \mathrm{mg} / \mathrm{m}^{2}$ cyclophosphamide on day $1,1.4 \mathrm{mg} / \mathrm{m}^{2}$

\footnotetext{
Received : February 4, 2013

Revised : April 30, 2013

Accepted : May 15, 2013

${ }^{1)}$ Department of Internal Medicine and Clinical Immunology, Yokohama City University Graduate School of Medicine, Yokohama, Japan

${ }^{2)}$ Department of Medical Oncology, Kanagawa Cancer Center, Yokohama, Japan

${ }^{3)}$ Department of Hematology, Yokohama City University Medical Center, Yokohama, Japan

${ }^{4}$ Department of Hematology/Immunology, Fujisawa City Hospital, Fujisawa, Japan

${ }^{5)}$ Department of Hematology, Shizuoka Red Cross Hospital, Shizuoka, Japan

${ }^{6}$ Department of Hematology, Yokosuka City Hospital, Yokosuka, Japan

${ }^{77}$ Department of Hematology, Yamato Municipal Hospital, Yamato, Japan

Corresponding author : Professor Naoto Tomita, Department of Internal Medicine and

Clinical Immunology, Yokohama City University Graduate School of Medicine, 3-9

Fukuura, Kanazawa-ku, Yokohama 236-0004, Japan

E-mail : cavalier@ch-yamate.dlenet.com
}

(maximum $2.0 \mathrm{mg} /$ body) vincristine on day $1,100 \mathrm{mg} /$ body of prednisolone on days $1-5$, and $375 \mathrm{mg} / \mathrm{m}^{2} \mathrm{R}$ per cycle] is currently one of the most frequently used regimens for DLBCL treatment and is one of the most common combination regimens used to treat FL. ${ }^{1,4}$ We therefore undertook a retrospective analysis of the outcomes of patients with FL or DLBCL who were uniformly treated using the standard RCHOP regimen to evaluate its benefit. The prognosis of grade $3 \mathrm{FL}$ was also evaluated and discussed.

\section{MATERIALS AND METHODS}

This study was approved by Yokohama City University Hospital Clinical Research Ethics Board. The procedures of this study were in accordance with the Helsinki Declaration. The Yokohama City University Hematology Group in Japan has uniformly and curatively treated patients with FL, except stage $1 \mathrm{FL}$, and DLBCL since 2001 and 2003, respectively, with 6 cycles of standard R-CHOP therapy for 21 days. We initiated R-CHOP therapy immediately after the diagnosis in patients with FL in stage 2-4 without a watchful waiting strategy. We collected the FL patients between 2001 and 2009, and DLBCL patients between 2003 and 2009. In these 
periods, 752 patients were treated (198, FL; 554, DLBCL), and 526 of these patients $(158, \mathrm{FL} ; 368, \mathrm{DLBCL})$ were enrolled in this study. The eligibility criteria for the analysis were patients with FL (stage 2-4) or DLBCL (any stages) who had initially been treated with full-dose R-CHOP therapy with curative intent. Patients who required more than $20 \%$ dose reduction were excluded. Actually, the most frequent cause of exclusion was dose reduction of the drugs. Those with special forms of DLBCL, such as intravascular lymphoma, primary mediastinal large B-cell lymphoma and T-cell-rich Bcell lymphoma, and those with human immunodeficiency virus infection were also excluded from this study. Although our study was not a prospective clinical study, we had registered newly diagnosed patients with lymphoma in our database 3 times a year, and the treatment protocol was fixed in advance. We therefore believe that our study resembles a prospective one to some degree. All included patients were scheduled to undergo primary therapy with 6 cycles of fulldose R-CHOP in 7 hospitals. Clinical staging was performed according to the Ann Arbor system, by using the data of physical examination; computed tomography of the neck, chest, abdomen and pelvis ; bone marrow aspiration and biopsy. When required, the data of endoscopic examination of the upper and/or lower gastrointestinal tract, lumbar puncture, magnetic resonance imaging of the brain, gallium scintigram and positron emission tomography were used for staging. Patients who had partial remission (PR) after the 4 initial cycles were administered 8 R-CHOP cycles in total, while patients who did not achieve PR after the 4 initial R-CHOP cycles or those who exhibited disease progression at any given time received salvage therapy. In these cases, the time point dealt with was that at disease progression. Additional local irradiation was also performed in patients with PR or complete remission (CR) if deemed necessary by the attending physician. No patients received maintenance therapy with R. Patients with DLBCL who achieved CR but were initially at risk of central nervous system (CNS) involvement also received methotrexate $(15 \mathrm{mg})$ and hydrocortisone $(25 \mathrm{mg}) 4$ times intrathecally for CNS prophylaxis. Central pathological reviews were not performed, and only individual institutional diagnoses according to the World Health Organization classification were used in subsequent analyses.

Univariate associations between histology (FL or DLBCL) and individual clinical features were analyzed using Chi-square test. The Mann-Whitney U test was used between ordered groups. Survival curves were compared using the log-rank test. A $P$-value below 0.05 was considered to indicate a significant difference.

\section{RESULTS}

Patient characteristics are shown in Table 1. In the FL group, 60 patients were at low risk (L); 60, at lowintermediate risk (LI) ; 26, at high-intermediate risk (HI) ;

Table 1. Patient characteristics

\begin{tabular}{|c|c|c|c|c|c|c|}
\hline \multirow{2}{*}{ Clinical data } & \multicolumn{4}{|c|}{ Follicular lymphoma (FL) } & \multirow{2}{*}{ DLBCL } & \multirow{2}{*}{$\begin{array}{c}\text { FL vs DLBCL } \\
p \text { value }\end{array}$} \\
\hline & All grades & Grade $1-2$ & Grade $3 a$ & Grade $3 b$ & & \\
\hline Number & 158 & 126 & 20 & 12 & 368 & \\
\hline Median age, years (range) & $57(25-76)$ & $57(25-76)$ & $59(34-72)$ & $50(32-70)$ & $64(18-80)$ & $<0.001$ \\
\hline Male $(\%)$ & 51 & 52 & 35 & 67 & 57 & NS \\
\hline \multicolumn{7}{|l|}{ IPI factors } \\
\hline Age greater that 60 years, $\%$ & 35 & 37 & 30 & 25 & 62 & $<0.001$ \\
\hline PS $2-4, \%$ & 3 & 4 & 5 & 0 & 15 & $<0.001$ \\
\hline Elevated LDH, \% & 30 & 26 & 50 & 42 & 49 & $<0.001$ \\
\hline Stage III/IV, \% & 80 & 82 & 70 & 75 & 43 & $<0.001$ \\
\hline More than 1 extranodal site, $\%$ & 37 & 36 & 35 & 50 & 29 & NS \\
\hline \multicolumn{7}{|l|}{ IPI } \\
\hline Low, \% & 38 & 38 & 35 & 42 & 43 & \\
\hline Low-intermediate, $\%$ & 38 & 41 & 35 & 17 & 25 & \\
\hline High-Intermediate, $\%$ & 16 & 14 & 20 & 33 & 16 & 0.005 \\
\hline High, \% & 8 & 7 & 10 & 8 & 16 & \\
\hline \multicolumn{7}{|l|}{ FLIPI* } \\
\hline Low, \% & 29 & 28 & 21 & 45 & NA & \\
\hline Intermediate, $\%$ & 32 & 35 & 32 & 10 & NA & \\
\hline High, \% & 39 & 37 & 47 & 45 & NA & \\
\hline
\end{tabular}

DLBCL, diffuse large B-cell lymphoma ; IPI, international prognostic index ; PS, performance status ; LDH, lactate dehydrogenase; FLIPI, follicular lymphoma international prognostic index.

*, FLIPI is not evaluable in 7 patients. 
and 12, at high risk $(\mathrm{H})$, as determined using the International Prognostic Index (IPI). ${ }^{5}$ According to the FL-IPI, ${ }^{6} 43$ patients were classified as L; 49, as intermediate risk; and 59, as $\mathrm{H}$. The risk of 7 patients was not determined. Ten patients in either PR or CR received additional local irradiation after RCHOP therapy, while none received CNS prophylaxis. Twelve deaths were observed among the FL patients during the observation period (median, 45 months in alive patients), 10 of which were due to the lymphomas. In the DLBCL group, 158 patients were classified as L ; 93, as LI ; 57 , as $\mathrm{HI}$; and 60 , as $\mathrm{H}$, according to the IPI, ${ }^{5}$ while 47 patients were classified as very good; 204, as good; and 117, as poor according to the R-IPI. ${ }^{7}$ Thirty-seven patients received additional local irradiation during PR and CR following the completion of R-CHOP therapy. CNS prophylaxis was performed in 42 patients who achieved CR and had an initial CNS risk. During the observation period (median, 43 months in alive patients), 58 DLBCL patients died, 50 of whom due to the lymphoma. In terms of the background, the patients older than 60 years $(P<0.001)$, with poor PS $(P<0.001)$ and with elevated LDH $(P<0.001)$ were more common in the DLBCL group. In contrast, the patients with advanced stage $(P<0.001)$ were more common in the FL group.

When the survival rates of the study participants were examined, it was found that the CR, 5-year progression-free survival (PFS) and 5-year overall survival (OS) rates of the FL and DLBCL groups were $86 \%, 50 \%$ and $92 \%$, and $89 \%$, $72 \%$ and $80 \%$, respectively. Interestingly, although the PFS rate of the DLBCL patients was significantly higher than that of patients with FL (Fig. 1A, $P=0.001$ ), patients in the FL group had a significantly greater OS (Fig. $1 \mathrm{~B}, P=0.006$ ). Furthermore, when the PFS (Fig. 1C) and OS (Fig. 1D) of patients with different grades of FL were compared, no significant differences between grade 1-2 FL and grade 3 FL were observed. This was also found to be the case when the PFS (Fig. 1E) and OS (Fig. 1F) of grade 1-3a FL patients were compared to those of grade 3b FL patients.

\section{DISCUSSION}

The findings of our study are similar to those of previous reports that examined the use of R-CHOP therapy in the treatment of $\mathrm{FL}^{1}$ and $\mathrm{DLBCL}^{7}$, including one that specifically examined the treatment of grade 3 FL. ${ }^{8}$ In our study, approximately $70 \%$ of the DLBCL patients who were treated with RCHOP therapy were cured, while the FL patients were generally incurable, albeit with the limitations of this study of its retrospective nature and lack of a pathological review. Furthermore, although patients with grade 1-2 and grade $3 \mathrm{FL}$ were found to have similar outcomes, this outcome was inferior to that obtained in DLBCL patients in terms of PFS. As for grade $3 \mathrm{FL}$, the PFS curve was apparently worse than that in DLBCL. Similar results were shown even in FL grade 3B patients, although it was difficult to evaluate the results because of the relatively small number of patients with FL grade 3B. These findings suggest the potential incurability of FL grade 3 patients by R-CHOP. This is a warning for clinicians who are likely to treat patients with grade $3 \mathrm{FL}$ as well as patients with DLBCL who have around $70 \%$ curability by RCHOP.

Various prognostic markers for DLBCL have been evaluated since the introduction of R. Bcl-2, a marker of the germinal center, has also been identified as a prognostic indicator of poor DLBCL outcome following CHOP therapy; however, it is possible to overcome resistance in DLBCL through the addition of R. ${ }^{9}$ Despite this, the addition of $\mathrm{R}$ to CHOP did not affect Bcl-6 protein expression, ${ }^{10}$ suggesting that the addition of R to CHOP may only be beneficial in Bcl6-negative DLBCL. This was also found in a study that focused on $B C L 6$ rearrangement. ${ }^{11}$ Furthermore, although the evaluation of these factors was not available in our multicenter retrospective study, we recently evaluated the prognostic value of $B C L 2$ and $B C L 6$ rearrangement for the efficacy of RCHOP therapy in patients with confirmed chromosomal abnormalities and found no correlation with the outcome. ${ }^{12}$ On the other hand, rearrangement of $M Y C$ is reported to be a definite poor prognostic marker ${ }^{13}$ and the evaluation of $M Y C$ is important in the R-era.

In conclusion, standard R-CHOP therapy is effective for patients with both FL and DLBCL, with the 5-year OS rate exceeding $80 \%$ for both groups. However, in the FL group, the PFS did not show a plateau, suggesting the incurability of this lymphoma with R-CHOP therapy. Despite this, the long OS observed suggests that salvage therapy is effective in the treatment of FL patients. This study also suggests that all grades of FL should be categorized the same in regards to RCHOP therapy since the OS and PFS in patients with grade 3 FL were similar to those in patients with grade 1-2 FL.

\section{CONFLICT OF INTEREST}

The authors declare that they have no conflicts of interest.

\section{REFERENCES}

1 Hiddemann W, Kneba M, Dreyling M, Schmitz N, Lengfelder E, et al.: Frontline therapy with rituximab added to the combination of cyclophosphamide, doxorubicin, vincristine, and prednisone (CHOP) significantly improves the outcome for patients with advanced-stage follicular lymphoma compared with therapy with CHOP alone: results of a prospective randomized study of the German Low-Grade Lymphoma Study Group. Blood 106:37253732, 2005

2 Pfreundschuh M, Trümper L, Osterborg A, Pettengell R, Trneny $\mathrm{M}$, et al.: CHOP-like chemotherapy plus rituximab versus CHOPlike chemotherapy alone in young patients with good-prognosis 

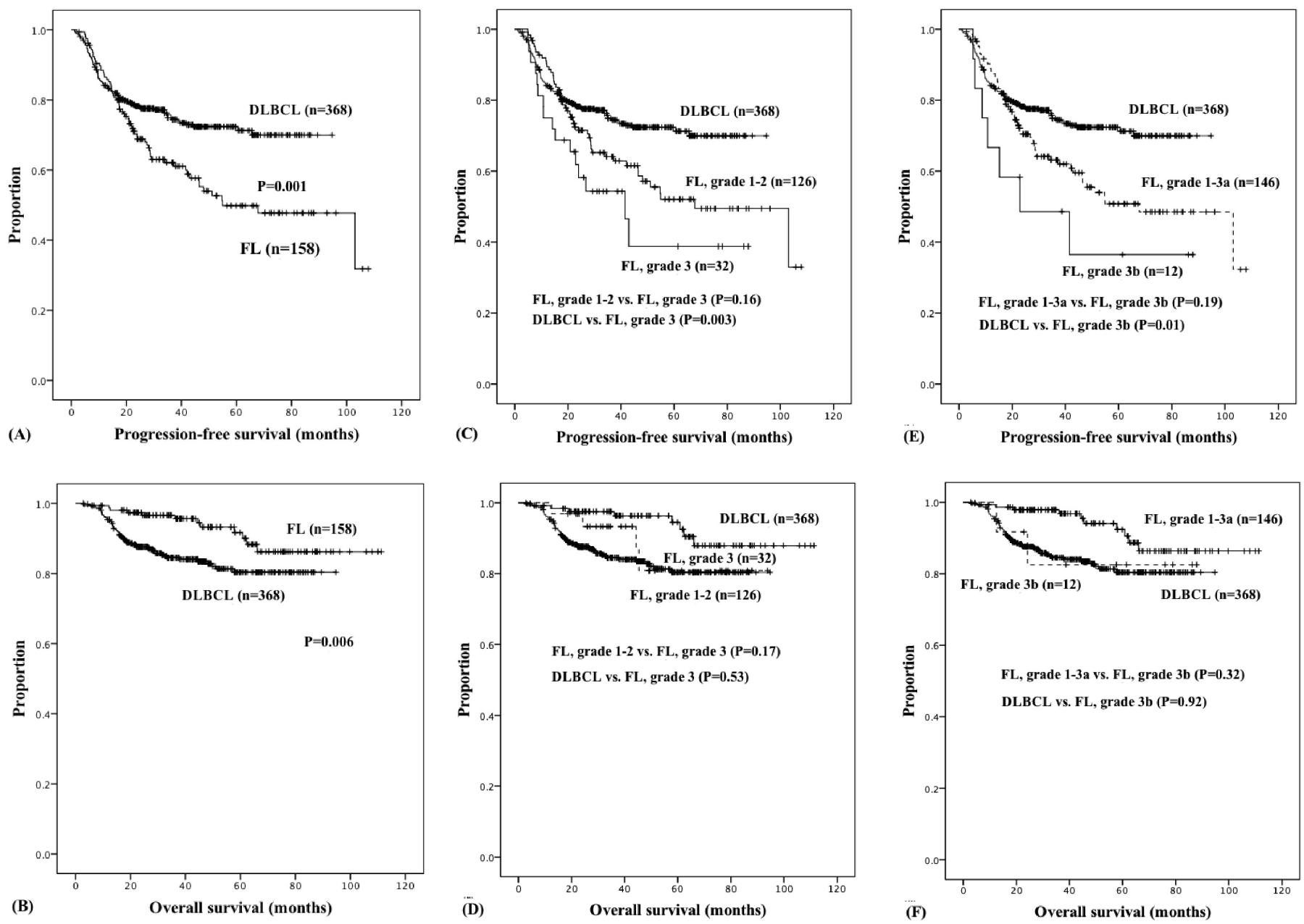

Fig. 1. The progression-free survival (PFS) and overall survival (OS) of follicular lymphoma (FL) and diffuse large B-cell lymphoma (DLBCL) patients following R-CHOP (rituximab, cyclophosphamide, doxorubicin, vincristine and prednisone) therapy. The PFS ( $1 A$ ) and OS $(1 B)$ of FL and DLBCL patients after the initiation of R-CHOP therapy demonstrated that PFS is significantly better in DLBCL patients, while the 5-year OS of FL patients, with a rate of $93 \%$, is significantly better than that of DLBCL patients. When only grade 3 FL patients are considered, the PFS $(1 C)$ of these patients is significantly worse than that of DLBCL, but the OS (ID) is not significantly different compared with that of grade 1-2 FL or DLBCL. This is also observed when the PFS $(1 E)$ and OS $(1 F)$ in grade $3 b$ FL patients are compared with those of either grade 1-3a FL or DLBCL patients.

diffuse large-B-cell lymphoma : a randomized controlled trial by the MabThera International Trial (MInT) Group. Lancet Oncol 7:379-391, 2006

3 Coiffier B, Thieblemont C, Van Den Neste E, Lepeu G, Plantier I, et al.: Long-term outcome of patients in the LNH-98.5 trial, the first randomized study comparing rituximab-CHOP to standard CHOP chemotherapy in DLBCL patients : a study by the Groupe d'Etudes des Lymphomes de l'Adulte. Blood 116:2040-2045, 2010

4 Siddhartha G, Vijay P: R-CHOP versus R-CVP in the treatment of follicular lymphoma : a meta-analysis and critical appraisal of current literature. J Hematol Oncol 2:14, 2009

5 No authors listed: A predictive model for aggressive nonHodgkin's lymphoma. The International Non-Hodgkin's
Lymphoma Prognostic Factors Project. N Engl J Med 329:987994, 1993

6 Buske C, Hoster E, Dreyling M, Hasford J, Unterhalt M, et al.: The Follicular Lymphoma International Prognostic Index (FLIPI) separates high-risk from intermediate- or low-risk patients with advanced-stage follicular lymphoma treated front-line with rituximab and the combination of cyclophosphamide, doxorubicin, vincristine, and prednisone (R-CHOP) with respect to treatment outcome. Blood 108:1504-1508, 2006

7 Sehn LH, Berry B, Chhanabhai M, Fitzgerald C, Gill K, et al.: The revised International Prognostic Index (R-IPI) is a better predictor of outcome than the standard IPI for patients with diffuse large B-cell lymphoma treated with R-CHOP. Blood 109:18571861,2007 
8 Shustik J, Quinn M, Connors JM, Gascoyne RD, Skinnider B, et al.: Follicular non-Hodgkin lymphoma grades $3 \mathrm{~A}$ and $3 \mathrm{~B}$ have a similar outcome and appear incurable with anthracycline-based therapy. Ann Oncol 22:1164-1169, 2011

9 Mounier N, Briere J, Gisselbrecht C, Emile JF, Lederlin P, et al.: Rituximab plus CHOP (R-CHOP) overcomes bcl-2-associated resistance to chemotherapy in elderly patients with diffuse large Bcell lymphoma (DLBCL). Blood 101:4279-4284, 2003

10 Winter JN, Weller EA, Horning SJ, Krajewska M, Variakojis D, et al.: Prognostic significance of Bcl-6 protein expression in DLBCL treated with $\mathrm{CHOP}$ or R-CHOP : a prospective correlative study. Blood 107:4207-4213, 2006
11 Shustik J, Han G, Farinha P, Johnson NA, Ben Neriah S, et al.: Correlations between BCL6 rearrangement and outcome in patients with diffuse large B-cell lymphoma treated with $\mathrm{CHOP}$ or R-CHOP. Haematologica 95:96-101, 2010

12 Watanabe R, Tomita N, Matsumoto C, Hattori Y, Matsuura S, et al. The $3 \mathrm{q} 27$ and $18 \mathrm{q} 21$ translocations for follicular lymphoma and diffuse large B-cell lymphoma in the rituximab era. J Clin Exp Hematop 107-114, 2013

13 Barrans S, Crouch S, Smith A, Turner K, Owen R, et al. Rearrangement of MYC is associated with poor prognosis in patients with diffuse large B-cell lymphoma treated in the era of rituximab. J Clin Oncol 28:3360-3365, 2010 